A N N A L E S Annales de Bretagne et des Pays de l'Ouest

\title{
The Pariahs of Yesterday. Breton Migrants in Paris
}

Jean Le Bihan

\section{OpenEdition}

Journals

Édition électronique

URL : http://journals.openedition.org/abpo/3170

DOI : $10.4000 / a b p o .3170$

ISBN : 978-2-7535-4882-4

ISSN : 2108-6443

Éditeur

Presses universitaires de Rennes

Édition imprimée

Date de publication : 15 décembre 2015

Pagination : 155-158

ISBN : 978-2-7535-4880-0

ISSN : 0399-0826

\section{Référence électronique}

Jean Le Bihan, «The Pariahs of Yesterday. Breton Migrants in Paris», Annales de Bretagne et des Pays de l'Ouest [En ligne], 122-4 | 2015, mis en ligne le 15 décembre 2015, consulté le 23 septembre 2020. URL : http://journals.openedition.org/abpo/3170 ; DOI : https://doi.org/10.4000/abpo.3170 
retrouve dans le commerce des vins, reconstituent parfaitement ce monde négociant dont les activités s'étendent du Bordelais à la Bretagne. Ce sont souvent les mêmes négociants qui contrôlent le commerce de la sardine pressée vers le Sud-Ouest ainsi que l'achat des vins dans le Bordelais, et chacun de ces deux secteurs bénéficie de la prospérité de l'autre. Le travail d'Hiroyasu Kimizuka nous fait également découvrir la strate intermédiaire du monde marchand qui investit dans l'armement, la construction navale, l'activité bancaire et même dans l'achat de cabarets. Le point sur les représentants de commerce fait connaître une profession très active à l'exemple d'un dénommé Toucas de Coussoles qui, tous les ans, fait la tournée de tous les grands ports importateurs bretons, des petits marchands locaux comme ceux de Châteaulin et se déplace même hors de la province jusqu'à Laval. Le dernier chapitre sur la ferme des devoirs s'attache à montrer les intérêts liés du monde du négoce et de la finance. On a affaire à un ensemble de capitalistes qui s'organisent pour réunir des capitaux, parfois jusqu'à Paris, dans le but d'accaparer les fermes et faire de juteux bénéfices. Ainsi Dutoya, sans doute le plus important négociant de Landerneau, personnage très riche qui prend en charge la régie des octrois de la ville. C'est aussi le monde de la fraude avec les changements de dénomination des vins, les fraudes sur les qualités, ses membres oubliant parfois de payer les droits qu'ils sont est censés prélever.

L'ouvrage d'Hiroyasu Kimizuka est original car il présente l'étude de la commercialisation d'un produit, le vin, à l'échelle d'une façade maritime, celle du golfe de Gascogne du fournisseur bordelais au consommateur breton. Cette recherche montrant le caractère fondamental des échanges agroalimentaires au cours de la période moderne est d'un grand apport à la connaissance de l'histoire du commerce breton mais aussi du petit cabotage européen.

Pierrick POURCHASSE

Moch, Leslie Page, The Pariahs of Yesterday. Breton Migrants in Paris, Durham, Duke University Press, 2012, 255 p.

La publication d'un ouvrage visant à réfléchir de manière globale sur l'histoire des Bretons installés à Paris et dans sa région entre la fin du XIX ${ }^{\mathrm{e}}$ siècle et la seconde moitié du Xx $x^{e}$ siècle constitue assurément une bonne nouvelle tant les travaux sur la question, s'ils n'étaient pas rares, demeuraient très dispersés, éclatés qu'ils étaient en trois principales catégories : les mémoires de maîtrise ou de master, les ouvrages d'érudition, enfin les études scientifiques plus larges portant soit sur l'émigration bretonne en général (au premier chef ceux de l'abbé Gautier qui, quelque discutables qu'ils soient à plusieurs égards, n'ont pas été remplacés à ce jour), soit sur l'immigration provinciale à Paris. On doit d'autant plus s'en réjouir que l'auteur, professeur à l'université d'État du Michigan, est une spécialiste reconnue de l'histoire des migrations européennes, ainsi qu'en témoigne, entre autres, son Moving Europeans. Migration in Western Europe since 1650 (Bloomington, Indiana University Press, $2^{\mathrm{e}}$ éd. 2003). De là vient d'ailleurs que The Pariahs nourrit en fait deux ambitions superposées : éclairer l'histoire propre, singulière, des Bretons de Paris - dimension qui retiendra prioritairement notre attention ici - mais aussi et au-delà alimenter, à partir de cette étude de cas, la réflexion plus générale sur le processus d'intégration nationale à l'œuvre en France sous la Troisième République. Il s'agit tout spécialement pour l'auteur de montrer la complexité de ce processus, l'histoire des Bretons de Paris fournissant à cet égard un très bon observatoire puisqu'il 
débute peu ou prou au moment où l'État républicain entreprend de mieux différencier les Français des étrangers et de soumettre les premiers au vaste programme de nationalisation que l'on sait. D'une certaine manière, on peut affirmer, qu'eu égard à ce problème fondamental, les Bretons de Paris se tiennent au départ dans une position ambiguë, au sens où ils sont à la fois dans la communauté nationale sur le plan politico-juridique et, en même temps, sinon hors, du moins en marge de cette même communauté sur le triple plan économique, social et culturel. Tout l'objet du livre est en somme de montrer comment cette tension va se défaire au profit d'une inclusion pleine et entière. Pour cela, L. P. Moch a d'abord fait l'effort d'assimiler la bibliographie existante; puis elle est allée aux sources : elle a abondamment utilisé les autobiographies disponibles, la presse du temps, enfin elle a dépouillé les actes de mariage contractés en 1890, 1910 et 1925 dans deux espaces tests connus pour avoir accueilli nombre de Bretons mais très dissemblables par ailleurs, savoir le quatorzième arrondissement de Paris et la commune de Saint-Denis. En tout, cinq chapitres : le premier, intitulé "Contextes ", plante en quelque sorte le décor, avant que les quatre autres ne s'enchaînent chronologiquement, traitant successivement des années 1880-1914, de l'entre-deux-guerres et des années 1945-1980.

Le mouvement d'ensemble que dessine l'ouvrage est donc celui de la progressive intégration des Bretons de la capitale à la société parisienne et, au-delà, d'une certaine manière, des Bretons tout court à la société française en général. Au départ, certes, les migrants bretons sont bien des parias, pour reprendre, comme l'auteur, ce mot saisissant utilisé dès 1898 par un ecclésiastique breton. C'est qu'en Bretagne, à la fin du XIX ${ }^{\mathrm{e}}$ siècle, la surpopulation des campagnes et la faiblesse du développement urbain se conjuguent pour jeter nombre de ruraux sur la route de Paris. Le département des Côtes-du-Nord, encore très ébranlé par l'effondrement de son industrie textile, constitue le principal pourvoyeur de ces exilés. La plupart sont sans qualification et bon nombre - les Bas-Bretons - sont en outre ignorants de la langue française. Beaucoup se font ouvriers ou bonnes. À cet afflux répond la présence accrue de la Bretagne dans les discours et les représentations dominants qui contribuent à la cristallisation d'un stéréotype dominé par l'idée d'arriération. Ce n'est pas un hasard, du reste, si c'est en 1905 que naît le personnage de Bécassine, la servante bretonne au grand cœur mais stupide. Cette époque est aussi celle des premiers efforts entrepris pour encadrer les Bretons de Paris. L'Église catholique joue en ce domaine un rôle primordial, comme en témoigne l'activité bien connue de La Paroisse bretonne. Créée en 1897 par l'énergique abbé Cadic, elle s'emploie à offrir toute une série de services et de secours aux Bretons fraîchement débarqués sur le pavé parisien tout en cherchant à fortifier leur ferveur religieuse et leur attachement à la Bretagne. Mais la situation évolue peu à peu durant l'entre-deux-guerres. Le flux des arrivées ne diminue certes pas et ce sont près de 130000 originaires des cinq départements de la Bretagne historique qui vivent à Paris au début des années 1930 . Les Finistériens sont à présent les plus nombreux, ce qui tient sans doute au fait que les campagnes cornouaillaises ont connu un maximum démographique particulièrement tardif. Des citadins se mêlent désormais aux ruraux, et, plus important, la proportion des Bretons qui accèdent à des emplois plus qualifiés, plus stables, source d'une modeste aisance, augmente. Trois autres facteurs jouent concomitamment dans le sens de leur plus grande intégration : l'engagement de nombre d'entre eux surtout des Dionysiens - dans les organisations politiques et syndicales de gauche, qui les dote d'une autre forme d'identité collective; leur différenciation croissante d'avec les nouveaux parias de l'heure que sont les étrangers, différenciation favorisée par les avantages réservés aux nationaux, notamment en matière d'accès à l'emploi; enfin l'affirmation, en leur sein, d'une élite culturelle prompte à s'insurger avec force contre les préjugés anti-bretons, lesquels d'ailleurs s'essoufflent, à l'image 
même de Bécassine dont l'épopée éditoriale prend fin juste avant la guerre. Est-ce la fin d'une époque? C'est ce que pense l'auteur, qui écrit sans détour : "1939 marked the close of the era of marginality for Bretons in Paris " (p. 157). De cette mutation témoigne d'une autre façon la perte d'influence de l'Église catholique, supplantée sur le terrain de la sociabilité régionale par la foule d'associations qui voient alors le jour et concilient de manière plutôt apaisée célébration de la petite patrie et dévotion à la grande. L'après-guerre fait plus que continuer cette dynamique, il l'accélère et en change même la nature. Étudiants et actifs qualifiés sont toujours plus nombreux parmi les migrants : transformation sociologique majeure, qui contribue grandement au délitement de la vie associative intra-bretonne à compter des années 1960 dans la mesure où la socialisation universitaire ou professionnelle devient pour beaucoup le vecteur principal d'intégration à la vie parisienne. Enfin, bien sûr, le renouveau culturel des années 1960 et 1970 confère à l'identité bretonne une positivité inédite sur fond de modernisation accélérée de la Bretagne en matière économique et culturelle. Mais ne développons pas plus : cette histoire est bien connue et le chapitre 5 est, comme le chapitre 1 , un essai de synthèse pour l'essentiel.

En définitive, ce qui nous paraît le plus neuf dans l'ouvrage de L. P. Moch, c'est l'utilisation qu'elle fait de l'état civil en vue de restituer la diversité des parcours individuels de tous ces terrassiers, domestiques, chauffeurs, employés de bureau et autres cafetiers bretons montés à Paris, de nous introduire, autant que possible, au cœur d'existences toujours singulières dont ne rendra jamais compte une histoire générale taillée à gros traits. Ainsi les deux terrains d'enquête retenus dévoilent-ils deux modèles très contrastés d'intégration au cours du demi-siècle décisif qui court des années 1880 aux années 1930. D'un côté Saint-Denis, la ville industrielle, où les hommes l'emportent longtemps en nombre et où les nouveaux-venus sont moins isolés qu'ailleurs du fait de la présence, sur place ou à proximité, d'une partie de leur famille. De l'autre le quatorzième, sis à l'ombre de Montparnasse, où les femmes sont légion, la propension à la promotion sociale plus grande, spécialement dans le secteur médical et les transports, et où les liens familiaux sont passablement distendus, ce qui fragilise certains déracinés. Notons qu'en 1925, l'endogamie, entendue comme le mariage entre deux individus originaires de la Bretagne historique, est à peu près de même importance dans les deux endroits (un petit tiers des unions), mais cela, assez curieusement, au terme d'une évolution qui l'a vue continument baisser à Saint-Denis et tout aussi continument augmenter dans le quatorzième arrondissement de Paris, évolution que, soit dit en passant, l'auteur interprète à tout coup comme la marque d'un surcroît d'intégration (p. 135, 142), ce qui mérite peut-être discussion. Ajoutons enfin que par-delà ce contraste territorial, fondamental, L. P. Moch se veut justement soucieuse de souligner l'ensemble des écarts et dénivellations qui font des Bretons de Paris une population éminemment hétérogène, en particulier ceux qui tiennent au genre (optique dont on sait l'importance au sein des études historiques anglo-saxonnes) ou à la position économique (avec raison, elle insiste sur le fait qu'à toute époque des cas de réussite individuelle sont observables parmi les Bretons établis dans la capitale ou sa banlieue).

On voit finalement tout le fruit d'une étude qui pense résolument le processus d'intégration en termes de trajectoires. Et l'on se prend à espérer que l'ouvrage de L. P. Moch suscite d'autres études appuyées sur la reconstitution fine des trajectoires individuelles et familiales des Bretons de Paris, que ce soit à partir de l'état civil, dont le dépouillement pourrait être mené à bien plus vaste échelle, des recensements de population, à l'image de ce qu'a déjà tenté, en pionnier, Marc Dutertre dans son mémoire de master (Bretons de Versailles. L'intégration des migrants au début du $X X^{e}$ siècle, mémoire de master 2, université de Versailles-Saint-Quentin-en-Yvelines, 2008) ou bien même des listes électorales. Pareille entreprise permettrait d'examiner 
en outre comment s'est poursuivie l'intégration à la deuxième génération, dimension fondamentale que L. P. Moch n'aborde pas. On ne peut en tout cas que recommander la lecture de ce livre stimulant, qui fera réfléchir et les historiens des migrations et les historiens de la Bretagne.

Jean LE BIHAN

Tison, Stéphane (dir.), Paul d'Estournelles de Constant. Concilier les nations pour éviter la guerre (1878-1924), Rennes, PUR, coll. « Histoire », 2015, 276 p.

La haute figure de Paul d'Estournelles de Constant (1852-1924) témoigne de la très grande qualité intellectuelle autant qu'humaine du personnel politique de la III République. Entré dans la carrière diplomatique en 1876, il s'illustre en Tunisie où, jeune diplomate, il est un des acteurs de l'élaboration du protectorat et du règlement des capitulations, puis à Londres. Conseiller d'ambassade auprès de l'ambassadeur William Waddington, il participe à l'instauration du protectorat à Madagascar et au règlement de la crise du Siam. Malgré ces débuts prometteurs, il quitte la carrière pour l'arène politique sans pour autant renoncer à son intérêt pour les affaires étrangères. Député de la Sarthe de 1895 à 1904, il est élu au Sénat de 1904 jusqu'à sa mort en 1924 où il siège à la Commission des Affaires étrangères.

C'est au sein de cette prestigieuse commission qu'il se montre un partisan infatigable de la paix dans une démarche qui, si elle n'est pas dénuée d'idéalisme, s'inscrit avant tout dans une démarche pragmatique. Observateur lucide de l'émergence de nouvelles puissances mondiales à l'orée du $\mathrm{xx}^{\mathrm{e}}$ siècle, il est animé par la volonté de préserver la paix dans un monde dont les hiérarchies que l'on pensait éternelles sont en train d'être remises en cause. À l'impossible retour en arrière comme à la crispation sur une suprématie fragile, d'Estournelles s'efforce de penser un monde nouveau. Conscient des fragilités du monde occidental, malgré l'imposant édifice impérial construit dans la seconde moitié du XIX ${ }^{e}$ siècle, et convaincu de l'inévitable émergence des périphéries du "monde fini ", il n'a de cesse d'imaginer le monde d'après. Il y a dans ce patriote un pessimiste qui prend acte de la fin annoncée de la domination européenne sur le monde et qui tente de penser les modalités de la survie du vieux continent dans un contexte où il ne peut plus prétendre à l'hégémonie. Convaincu de la menace mortelle que représentait, pour cette survie, une nouvelle guerre, il participe largement à l'élaboration d'un droit international, par sa présence à la conférence de La Haye de 1899 où il contribue à sauver le principe de la cour d'arbitrage, dont il réussit à renforcer les pouvoirs lors de la conférence de 1907. Il se montre par ailleurs un analyste perspicace des premiers effets de la mondialisation économique; convaincu de l'aspect inévitable de ce mouvement, il cherche à penser les cadres, économiques et politiques, susceptibles d'offrir la meilleure protection à une Europe dont il sait, sous la puissance apparente, la fragilité.

Les nombreuses critiques, en raison de son incessant plaidoyer pour la paix, couronné par la remise du prix Nobel en 1909, qui ne voient en lui qu'un utopiste, ne sont pas sans préfigurer celles qui accueillent l'action politique de Briand dans les années 1920. À bien des égards, les deux hommes partagent un même pessimisme, nourri d'une lecture sans illusion de la situation internationale. L'affaiblissement de l'Europe, son essoufflement économique, la menace permanente de la guerre, sont des réalités difficilement contestables. Maintenir une hiérarchie mondiale autour du souvenir de la puissance relève d'une illusion dangereuse dont la poursuite est promesse de nouveaux conflits. Si d'Estournelles ne propose pas de solution à cette 